\title{
BMJ Open Medical service use and usual care of common shoulder disorders in Korea: a cross-sectional study using the Health Insurance Review and Assessment Service National Patient Sample
}

\author{
Hwansoo Joo, ${ }^{1}$ Yoon Jae Lee, ${ }^{1}$ Joon-Shik Shin, ${ }^{1}$ Jinho Lee, ${ }^{1}$ Me-riong Kim, ${ }^{1}$ \\ Wonil Koh, ${ }^{1}$ Yeoncheol Park, ${ }^{2}$ Yun Kyung Song, ${ }^{3}$ Jae-Heung Cho, ${ }^{4}$ In-Hyuk Ha ${ }^{1}$
}

To cite: Joo H, Lee YJ, Shin J-S, et al. Medical service use and usual care of common shoulder disorders in Korea: a cross-sectional study using the Health Insurance Review and Assessment Service National Patient Sample. BMJ Open 2017;7:e015848. doi:10.1136/ bmjopen-2017-015848

- Prepublication history and additional material are available. To view these files please visit the journal online (http://dx.doi org/10.1136/bmjopen-2017015848).

HJ and YJL contributed equally.

Received 4 January 2017

Revised 3 June 2017

Accepted 9 June 2017

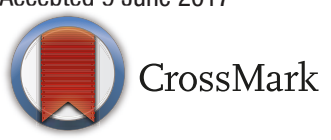

${ }^{1}$ Jaseng Spine and Joint Research Institute, Jaseng Medical Foundation, Seoul, The Republic of Korea

${ }^{2}$ Department of Acupuncture and Moxibustion, Kyung Hee University Hospital at Gangdong, Seoul, The Republic of Korea ${ }^{3}$ Department of Korean Rehabilitation Medicine, College of Korean Medicine, Gachon University, Seongnam-si, The Republic of Korea

${ }^{4}$ Department of Korean Rehabilitation Medicine, College of Korean Medicine, Kyung Hee University, Dongdaemun-gu, Seoul, The Republic of Korea

Correspondence to

Dr. In-Hyuk Ha; hanihata@gmail. com

\section{ABSTRACT}

Objectives This study examined National Health Insurance claims data to investigate the epidemiology of shoulder disorders in Korea. Detailed information on medical services and related costs was assessed by major shoulder disorder category.

Design and setting The 2014 National Patient Sample dataset provided by the Health Insurance Review and Assessment Service was analysed. Among shoulderrelated diagnosis codes, adhesive capsulitis of the shoulder (ACS), rotator cuff syndrome (RCS) and shoulder impingement syndrome (SIS) categories were of highest prevalence. Sociodemographic characteristics and medical service use, frequency and medical costs regarding common shoulder disorders were evaluated.

Results The majority of patients with shoulder disorder received ambulatory care (97\%). Total and per-patient expenses were highest in patients with RCS. The number of inpatients with RCS was more than twice that of the other two groups, and patients with RCS were more likely to receive surgical management compared with patients with ACS and SIS. Prevalence of shoulder disorders was highest among subjects in their 50s for all three groups. Primary care physicians treated $75.80 \%$ of patients with ACS, $56.99 \%$ of patients with RCS and $48.06 \%$ of patients with SIS, respectively, outlining the difference in medical institution usage patterns. In all three groups, the highest proportion of patients visited orthopaedic surgeons out of medical departments. In the ACS and SIS groups, cost of visits (consultations) took up the largest part of total expenses at $32.30 \%$ and $18.88 \%$, respectively, while cost of procedure/surgery constituted the largest portion in patients with RCS $(37.77 \%)$. The usage proportion of subcutaneous or intramuscular and intra-articular injections ranged between $20 \%$ and $30 \%$ for outpatients in all three groups.

Conclusions Medical service use, frequency and cost distributions relating to major shoulder disorders in Korea were assessed using nationwide claims data. These findings are expected to aid policy-makers as well as researchers and practitioners as basic healthcare data.
Strengths and limitations of this study

- Approximately $97 \%$ of the South Korean population is covered by National Health Insurance Service, and this study effectively uses the claims database to examine and compare medical use and costs of high-frequency shoulder disorders in a nationally representative sample.

- This study employs national healthcare data to provide a detailed and current perspective of prevalence, medical expenditure and usual care in surgery, injections, physiotherapy and medication use of major shoulder lesions and to provide basic usage data relevant for health policy implementation and budget appropriation.

- While data are efficiently collected and managed by the Korean Health Insurance Review and Assessment Service through the claims review process, items not covered by National Health Insurance such as overthe-counter drugs and out-of-pocket expenditures could not be assessed.

- In addition, due to the fact that analysis was performed based on administrative codes of frequent shoulder disorders as opposed to clinical conditions or symptoms, the possibility of mismatched coding cannot be ruled out.

- Still, the authors attempted to enhance internal validity by collecting data from patients with $\mathrm{X}$-ray results.

\section{INTRODUCTION}

Shoulder pain is commonly seen in primary healthcare and incurs significant pain and disability, healthcare costs and work loss. ${ }^{1}$ The annual prevalence and incidence of patients with shoulder disorder in UK primary care were $2.36 \%$ and $1.47 \%$, respectively, ${ }^{2}$ and its annual prevalence in Dutch primary care was estimated to be about 11 per 1000 patients. ${ }^{3}$ A 2004 systematic review reported point prevalence of $6.9 \%-26 \%$ and lifetime prevalence 
of $6.7 \%-66.7 \%$ with higher estimates gauged from older study populations (65+ years). ${ }^{4}$ Meanwhile, 2011 Korean National Health Insurance Service statistics showed that a total $4.3 \%$ of the Korean population was diagnosed with shoulder pain (Korean Standard Classification of Diseases (KCD) diagnosis code M75) with an average annual increase of $8.9 \%$ over the past 6 years, indicating that the importance of shoulder pain diagnosis and treatment is growing in primary and national healthcare settings. ${ }^{5}$

Shoulder pain is the third most common cause of primary care consultation in musculoskeletal conditions, and approximately $1 \%$ of UK adults annually consult a general practitioner for new shoulder pain according to a 1998 population survey of 6000 UK clinic patients. ${ }^{6}$ Annual prevalence estimates of shoulder pain and medical consultations in Australian general practice have also been put at $1 \%-1.5 \%{ }^{7}$ While chronic shoulder pain is a common manifestation, its pathogenesis is often diverse and complex, incurring substantial medical costs in diagnosis and treatment, and shoulder problems are a significant cause of morbidity and disability in the general population. ${ }^{8}$ In 1995 alone, musculoskeletal disorders accounted for 9.9 million days of sick leaves in the UK of which 4.2 million (42\%) were related to the upper limb and neck area, ${ }^{9}$ showing that musculoskeletal complaints place a heavy burden on primary care services in general ${ }^{2}$ and that the economic burden of upper limb disorders is substantial. ${ }^{8}$ Direct medical expenses for shoulder disability reached US $\$ 7$ billion in the USA in $2000 .{ }^{10}$ Similarly, in Korea, 1954642 patients were diagnosed with shoulder disorders (KCD, sixth revision (KCD-6) diagnosis code M75) with an annual health expenditure of approximately US $\$ 39.8$ million according to the 2014 National Health Insurance Statistical Yearbook. ${ }^{11}$

Chronic shoulder pain has been linked with various pathologies ranging from bursitis, tendinitis, rotator cuff tear, adhesive capsulitis, impingement syndrome, avascular necrosis and glenohumeral osteoarthritis (OA) to joint degeneration and injury, which can occur either singularly or in combination. Rotator cuff injury and adhesive capsulitis hold high prevalence of chronic disorders, comprising $10 \%$ and $6 \%$ of all shoulder-related disorders, respectively. ${ }^{10}$ Shoulder impingement syndrome is also common with 5 out of 1000 individuals annually diagnosed as new clinical cases. ${ }^{3}$ However, disproportionate to the high prevalence and associated costs of adhesive capsulitis, rotator cuff syndrome and shoulder impingement syndrome, comprehensive studies detailing specific components of usual care and related medical expenses have been found lacking.

The Korean healthcare system is implemented under the National Health Insurance programme, which enforces legal obligation and authority as a universal social insurance programme covering approximately $97 \%$ of the South Korean population. The National Health Insurance Service (NHIS) operates the healthcare system under government supervision and promotes public health by providing basic healthcare services. ${ }^{12}$ This study aimed to assess the prevalence, associated healthcare use and related costs of common shoulder disorders in Korea through analysis of National Health Insurance claims data provided by the Health Insurance Review and Assessment Service (HIRA), which reviews and assesses all NHIS claims data. This dataset includes nationally representative data of 1.4 million patients, covering approximately $3 \%$ of 47 million Koreans covered by National Health Insurance out of the total South Korean population (51 million).

\section{METHODS}

\section{Participants}

The 2014 National Patient Sample (NPS) dataset analysed in this study was provided by HIRA. Details on specific medical services and prescription history for the past year, as well as the demographic characteristics from a $3 \%$ sample randomly extracted after stratification by age (in 5-year intervals) and sex, are provided by HIRA on an annual basis for the aim of enhancing data accessibility and convenience of researchers. This secondary dataset is systematically sampled through stratification after removing identifying personal and institutional information from the raw claims data of all provided medical services compiled from all patients with medical service use for the past year as of the medical care commencement date of the corresponding year. The NPS sample dataset contains information on approximately 1.4 million patients. $^{13}$

\section{Shoulder disorders}

Data were collected from all patients who were diagnosed with subcategory codes M75 (collective referral to all subcategory codes under basic KCD-6 code 'M75,' for shoulder disorders) as the primary diagnosis and had shoulder X-ray results taken within the corresponding year. Adhesive capsulitis of the shoulder (ACS; KCD-6 code M750), rotator cuff tear or rupture (RCS; KCD-6 code M751) and shoulder impingement syndrome (SIS; KCD-6 code M754) were included as the major categories of shoulder disorders based on prevalence. A total of 10336, 9387 and 7135 outpatients and 464, 1432 and 695 inpatients were diagnosed with ACS (total $\mathrm{n}=10447$ ), RCS (total $\mathrm{n}=9689$ ) and SIS (total $\mathrm{n}=7328$ ), respectively. The total number of patients in each subset may be smaller than the numerical sum of corresponding inpatients and outpatients as some patients received both ambulatory and hospitalised care, and tallies allowed for duplicates. All shoulder-related diagnostic codes and numbers of patients are summarised in online supplementary table S1.

\section{Analysis}

Sociodemographic characteristics of patients investigated in this study included age, sex, insurance eligibility, type of medical institution visited for medical service and specialty of consulting physicians. Medical institutions were classified into primary care clinics, hospitals, general 
hospitals, tertiary hospitals, long-term care hospitals and public health centres. Hospitals hold $\geq 30$ inpatient beds, while general hospitals operate $\geq 300$ beds for inpatient care and at least three outpatient departments out of internal medicine, surgery, paediatrics, and obstetrics and gynaecology with corresponding full-time specialists. Tertiary hospitals provide specialised, advanced medical care for serious medical disorders out of general hospitals with $\geq 20$ departments designated by order of the Korean Ministry of Health and Welfare with corresponding fulltime specialists for each specialty. ${ }^{14}$

Medical service costs covering costs for visit (consultation), hospitalisation, medication, injection, anaesthesia, physiotherapy, psychotherapy, procedure/surgery, examination and radiologic evaluation/intervention were calculated from corresponding codes as designated according to Ministry of Health and Welfare notification. Total medical expense was determined as the sum of co-payment paid by the patient and insurance benefit covered by the National Health Insurance, of which the benefit amount is determined through HIRA review. Frequency of medical service use, including surgery, injection, physiotherapy and analgesics, was investigated in inpatients and outpatients separately and totalled for each diagnosis category. The codes for surgery, injection and physiotherapy are given in online supplementary table S2, and detailed information on each code is available online as a guidebook in PDF format from the HIRA website. ${ }^{15}$ Only service codes that were verified to be relevant to shoulder disorders were analysed. Physiotherapy and rehabilitation therapy types prescribed to at least 500 cases were assessed for frequency. Drugs prescribed in inpatient or outpatient departments were classified according to Anatomical Therapeutic Chemical (ATC) Classification System fourth-level classifications and were organised in order of usage proportion by non-narcotic and narcotic categories. The non-narcotic and narcotic drug classifications were adopted from analgesics categories in a previous report from the Korean National Evidence-based Healthcare Collaborating Agency. ${ }^{16}$ Although most drug substance names are identical with ATC fifth-level classifications, which indicate chemical names, a few exceptions do not have matching classifications. A drug was considered to be prescribed following medication code records, regardless of dose.

\section{Statistical analysis}

All statistical analyses were performed using SAS version 9.3 (SAS Institute, Cary, North Carolina, USA). The sociodemographic characteristics and medical services provided to inpatients and outpatients were analysed through frequency analysis for each shoulder disorder category. Descriptive analysis results are presented as frequencies and percentages. Percentages were calculated with total number of patients or total expense in the corresponding disease group set as the denominator. Total expense and per-patient expenses for each disease group were also calculated.

\section{Ethics statement}

This study was reviewed and approved by the Institutional Review Board of Jaseng Hospital of Korean Medicine (JASENG 2016-11-002).

\section{RESULTS}

\section{Medical service use for ACS, RCS and SIS}

While the ACS group contained the highest total number of patients $(\mathrm{n}=10447)$, total expense, per-patient expense, average days of care and average numbers of visits were highest in the RCS group. Outpatient values were highest in the ACS group, followed by RCS and SIS groups in number of patients, total expense, per-patient expense, average days of care and average numbers of visits, while inpatient values were highest in the RCS group, followed by the SIS and ACS groups. Total medical expenditure was $56.84 \%$ in inpatients $(9.43 \%)$ compared with $43.16 \%$ in outpatients $(90.57 \%)$, even though the majority of patients were outpatients (table 1).

\section{Characteristics of ACS, RCS and SIS patients}

The prevalence of ACS, RCS and SIS was highest among patients in their $50 \mathrm{~s}$, and women were more likely to be affected than men. About $97 \%$ of all patients received medical care in the ambulatory department and $96 \%$ were National Health Insurance-eligible. The most frequently visited medical institution types were primary care clinics, followed by hospitals and general hospitals. The proportion of visits to primary care clinics in ACS cases was $75.80 \%$, which was higher than in the other diagnosis groups $(56.99 \%$ in RCS and $48.06 \%$ in SIS, respectively). Patients were treated by orthopaedic surgeons in $80 \%-90 \%$ of all cases regardless of shoulder disorder type (table 2 ).

\section{Distribution of ACS, RCS and SIS-related costs}

All medical expenses were assessed according to the type of medical service provided (ie, visit (consultation), hospitalisation, injection, anaesthesia, physiotherapy, psychotherapy, procedure/surgery, examination and radiologic evaluation/intervention). Costs of visit (consultation), anaesthesia and physiotherapy took up a significant portion of ACS costs, whereas costs of procedure/surgery, hospitalisation and visits (consultations) were dominant for patients with RCS, and costs of procedure/surgery, visits (consultations) and hospitalisation for patients with SIS. The sum of costs for procedure/ surgery and hospitalisation were $53.17 \%$ and $37.24 \%$ of total expenses for patients with RCS and SIS, respectively, while the sum was low at $6.46 \%$ in patients with ACS. In contrast, the cost of injections was higher accounting for $11.46 \%$ of the total expense in patients with ACS, compared with those of $6.94 \%$ and $8.60 \%$ in patients with RCS and SIS, respectively. More than $10 \%$ of all patients were prescribed drugs, but the cost of medication amounted to less than $2 \%$ of total medical expense in all three groups. Percentages of physiotherapy cost out of total medical expense were $61.79 \%, 53.72 \%$ and 
Table 1 General medical service use for adhesive capsulitis of the shoulder, rotator cuff syndrome and shoulder impingement syndrome in Korea

\begin{tabular}{|c|c|c|c|c|c|c|}
\hline Visit type & $\begin{array}{l}\text { Disorder } \\
\text { groups }\end{array}$ & Patients (n) & Total expense* & $\begin{array}{l}\text { Per-patient } \\
\text { expense* }\end{array}$ & $\begin{array}{l}\text { Days of } \\
\text { treatment }+\end{array}$ & Visits (n)‡ \\
\hline \multirow[t]{3}{*}{ Total } & ACS & 10447 & $\begin{array}{l}1817540220 \\
(1588758.9)\end{array}$ & $\begin{array}{l}173977.2 \\
(152.1)\end{array}$ & 6.3 & 5.7 \\
\hline & RCS & 9689 & $\begin{array}{l}4147531010 \\
(3625464.2)\end{array}$ & $\begin{array}{l}428066.0 \\
(374.2)\end{array}$ & 8.4 & 6.1 \\
\hline & SIS & 7328 & $\begin{array}{l}1621638960 \\
(1417516.6)\end{array}$ & $\begin{array}{l}221293.5 \\
(193.4)\end{array}$ & 5.8 & 4.6 \\
\hline \multirow[t]{3}{*}{ Outpatient } & ACS & 10336 & $\begin{array}{l}1415669040 \\
(1237472.9)\end{array}$ & $\begin{array}{l}136964.9 \\
(119.7)\end{array}$ & 5.7 & 5.4 \\
\hline & RCS & 9387 & $\begin{array}{l}1144119540 \\
(1000104.5)\end{array}$ & $\begin{array}{l}121883.4 \\
(106.5)\end{array}$ & 5.4 & 4.7 \\
\hline & SIS & 7135 & $\begin{array}{l}714434250 \\
(624505.5)\end{array}$ & $\begin{array}{l}100130.9 \\
(87.5)\end{array}$ & 4.2 & 3.9 \\
\hline \multirow[t]{3}{*}{ Inpatient } & ACS & 464 & $\begin{array}{l}401871180 \\
(351286.0)\end{array}$ & $\begin{array}{l}866101.7 \\
(757.1)\end{array}$ & 15.2 & 6.6 \\
\hline & RCS & 1432 & $\begin{array}{l}3003411470 \\
(2625359.7)\end{array}$ & $\begin{array}{l}2097354.4 \\
(1833.4)\end{array}$ & 21.4 & 10.7 \\
\hline & SIS & 695 & $\begin{array}{l}907204710 \\
(793011.1)\end{array}$ & $\begin{array}{l}1305330.5 \\
(1141.0)\end{array}$ & 17.5 & 8.5 \\
\hline
\end{tabular}

*Displayed in KRW; US\$1=1144 KRW (as of October 31, 2016); approximate US\$ equivalents are given beneath in parentheses.

†Total days of treatment per patient as indicated in the claims statement, which includes drug prescription days without medical treatment. $\ddagger$ Total number of visits or number of hospitalised days per patient as indicated in the claims statement.

ACS, adhesive capsulitis of the shoulder; RCS, rotator cuff syndrome; SIS, shoulder impingement syndrome.

$52.46 \%$ in the ACS, RCS and SIS groups, respectively, and more than half of all patients received physiotherapy in each disease category. The cost of radiologic evaluation/ intervention totalled to less than $10 \%$ of total expense, and the proportion of injection use was higher than that of drug prescription for all the three groups (table 3).

\section{Usual care for ACS, RCS and SIS}

The percentage of surgically treated cases was highest in the RCS group and lowest in the ACS group. The most frequently performed surgery for shoulder disorders was acromioplasty. The proportion of subcutaneous or intramuscular and intra-articular injections in outpatients was comparable in all three groups, ranging from $20 \%$ to $30 \%$, although the proportion was slightly higher in the ACS group. In order of decreasing frequency, superficial heat therapy, deep heat therapy, transcutaneous electrical nerve stimulation and interferential current therapy were the most commonly used physiotherapy modalities, and overall prescription proportion of physiotherapies was highest in the ACS group, followed by the RCS and SIS groups. Heat therapies including superficial and deep heat therapies were prescribed to about half of all shoulder cases. The proportions of transcutaneous electrical nerve stimulation and interferential current therapy ranged from $20 \%$ to $30 \%$ in all three groups. Meanwhile, the prescription proportion of laser therapy was less than $10 \%$.
Simple therapeutic exercise was prescribed to $19.04 \%$ of patients with ACS, which was more than twice that of the RCS and SIS groups; however, complex therapeutic exercise was prescribed more often in the RCS group $(7.05 \%)$ than in the ACS $(2.90 \%)$ or SIS $(4.49 \%)$ groups. Other physiotherapies were less frequently prescribed, but it is worth noting that the proportion of complex therapeutic exercise was significantly higher in inpatients than in outpatients at $23.06 \%, 27.58 \%$ and $29.78 \%$ in the ACS, RCS and SIS groups, respectively. Myofascial trigger point injection therapy was seldom used in both inpatients and outpatients (table 4).

\section{Medication use in ACS, RCS and SIS}

The drugs prescribed in shoulder disorders were classified according to the ATC fourth level. The absolute majority of drug prescriptions were non-narcotic ( $\mathrm{n}=35021 / 36638$; $95.38 \%)$, and narcotic analgesics, when used, were mainly prescribed in hospital settings ( $\mathrm{n}=1505 / 1617 ; 93.07 \%)$. Among non-narcotic drugs, acetic acid derivatives and related substances, other opioids, propionic acid derivatives, other anti-inflammatory and anti-rheumatic agents (non-steroids), oxicams and anilide groups were frequently prescribed (listed in order of frequency). Tramadol was the only drug prescribed from the other opioids group. Of narcotic drugs, opioid anaesthetics, phenylpiperidine derivatives and natural opium alkaloids were commonly prescribed (listed in order of frequency) (table 5). 
Table 2 Sociodemographic characteristics of adhesive capsulitis of patients with shoulder, rotator cuff syndrome and shoulder impingement syndrome

\begin{tabular}{|c|c|c|c|c|c|c|c|c|}
\hline \multirow[b]{2}{*}{ Characteristics } & \multicolumn{2}{|l|}{ ACS } & \multicolumn{2}{|l|}{ RCS } & \multicolumn{2}{|l|}{ SIS } & \multicolumn{2}{|c|}{ Total sample population } \\
\hline & $n=10447$ & $\%$ & $\mathrm{n}=9689$ & $\%$ & $n=7328$ & $\%$ & $n=1438178$ & $\%$ \\
\hline \multicolumn{9}{|l|}{ Age (years) } \\
\hline$<20$ & 21 & 0.20 & 80 & 0.83 & 70 & 0.96 & 313646 & 21.81 \\
\hline 20 29 & 91 & 0.87 & 263 & 2.71 & 278 & 3.79 & 180111 & 12.52 \\
\hline 30 39 & 337 & 3.23 & 679 & 7.01 & 662 & 9.03 & 217735 & 15.14 \\
\hline $40 \sim 49$ & 1838 & 17.59 & 1925 & 19.87 & 1731 & 23.62 & 242157 & 16.84 \\
\hline $50 \sim 59$ & 3761 & 36.00 & 3186 & 32.88 & 2508 & 34.22 & 228492 & 15.89 \\
\hline $60 \sim 69$ & 2465 & 23.60 & 2160 & 22.29 & 1304 & 17.79 & 131499 & 9.14 \\
\hline$\geq 70$ & 1934 & 18.51 & 1396 & 14.41 & 775 & 10.58 & 124538 & 8.66 \\
\hline \multicolumn{9}{|l|}{ Sex } \\
\hline Male & 4178 & 39.99 & 4348 & 44.88 & 3471 & 47.37 & 699035 & 48.61 \\
\hline Female & 6269 & 60.01 & 5341 & 55.12 & 3857 & 52.63 & 739143 & 51.39 \\
\hline \multicolumn{9}{|l|}{ Visit type } \\
\hline Inpatient & 464 & 4.44 & 1432 & 14.78 & 695 & 9.48 & 193184 & 13.43 \\
\hline Outpatient & 10336 & 98.94 & 9387 & 96.88 & 7135 & 97.37 & 1433858 & 99.70 \\
\hline \multicolumn{9}{|l|}{ Insurance type } \\
\hline National Health Insurance & 9984 & 95.57 & 9336 & 96.36 & 7092 & 96.78 & 1398282 & 97.23 \\
\hline Medicaid & 470 & 4.50 & 356 & 3.67 & 235 & 3.21 & 44652 & 3.11 \\
\hline Veteran healthcare & 9 & 0.09 & 32 & 0.33 & 18 & 0.25 & 3260 & 0.23 \\
\hline \multicolumn{9}{|l|}{ Medical institution } \\
\hline Clinic & 7919 & 75.80 & 5522 & 56.99 & 3522 & 48.06 & 1316958 & 91.57 \\
\hline Hospital & 2243 & 21.47 & 2786 & 28.75 & 3020 & 41.21 & 406611 & 28.27 \\
\hline General hospital & 741 & 7.09 & 1473 & 15.20 & 945 & 12.90 & 375571 & 26.11 \\
\hline Tertiary hospital & 138 & 1.32 & 648 & 6.69 & 157 & 2.14 & 207937 & 14.46 \\
\hline Long-term care hospital & 87 & 0.83 & 31 & 0.32 & 21 & 0.29 & 27437 & 1.91 \\
\hline Public health centre & 41 & 0.39 & 18 & 0.19 & 4 & 0.05 & 81007 & 5.63 \\
\hline \multicolumn{9}{|l|}{ Medical specialty } \\
\hline Orthopaedic surgery & 8762 & 83.87 & 8670 & 89.48 & 6737 & 91.94 & 584569 & 40.65 \\
\hline Anaesthesiology & 750 & 7.18 & 560 & 5.78 & 334 & 4.56 & 58270 & 4.05 \\
\hline Neurosurgery & 490 & 4.69 & 261 & 2.69 & 142 & 1.94 & 107990 & 7.51 \\
\hline Rehabilitation medicine & 483 & 4.62 & 374 & 3.86 & 181 & 2.47 & 33104 & 2.30 \\
\hline General surgery & 352 & 3.37 & 100 & 1.03 & 65 & 0.89 & 222058 & 15.44 \\
\hline Internal medicine & 240 & 2.30 & 81 & 0.84 & 42 & 0.57 & 966320 & 67.19 \\
\hline Family medicine & 192 & 1.84 & 73 & 0.75 & 53 & 0.72 & 152569 & 10.61 \\
\hline Other & 95 & 0.91 & 24 & 0.25 & 13 & 0.18 & 875225 & 60.86 \\
\hline General physician & 33 & 0.32 & 9 & 0.09 & 1 & 0.01 & 82475 & 5.73 \\
\hline
\end{tabular}

*Includes neurology, radiology, emergency medicine, occupational and environmental medicine, neuropsychiatry, paediatrics, thoracic and cardiovascular surgery, obstetrics and gynaecology, and urology.

ACS, adhesive capsulitis of the shoulder; RCS, rotator cuff syndrome; SIS, shoulder impingement syndrome.

Prescription details in accordance with fifth-level ACT codes are summarised in online supplementary table S3.

\section{DISCUSSION}

The prevalence and medical service use of high-prevalence shoulder disorders in Korea were investigated based on 2014 HIRA-NPS data, and treatment types and related costs were further analysed. In all three groups, the outpatient proportion was higher. Shoulder disorders were most prevalent in middle-aged populations. ACS cases were more often treated in primary care settings than RCS and SIS, but a high percentage of 


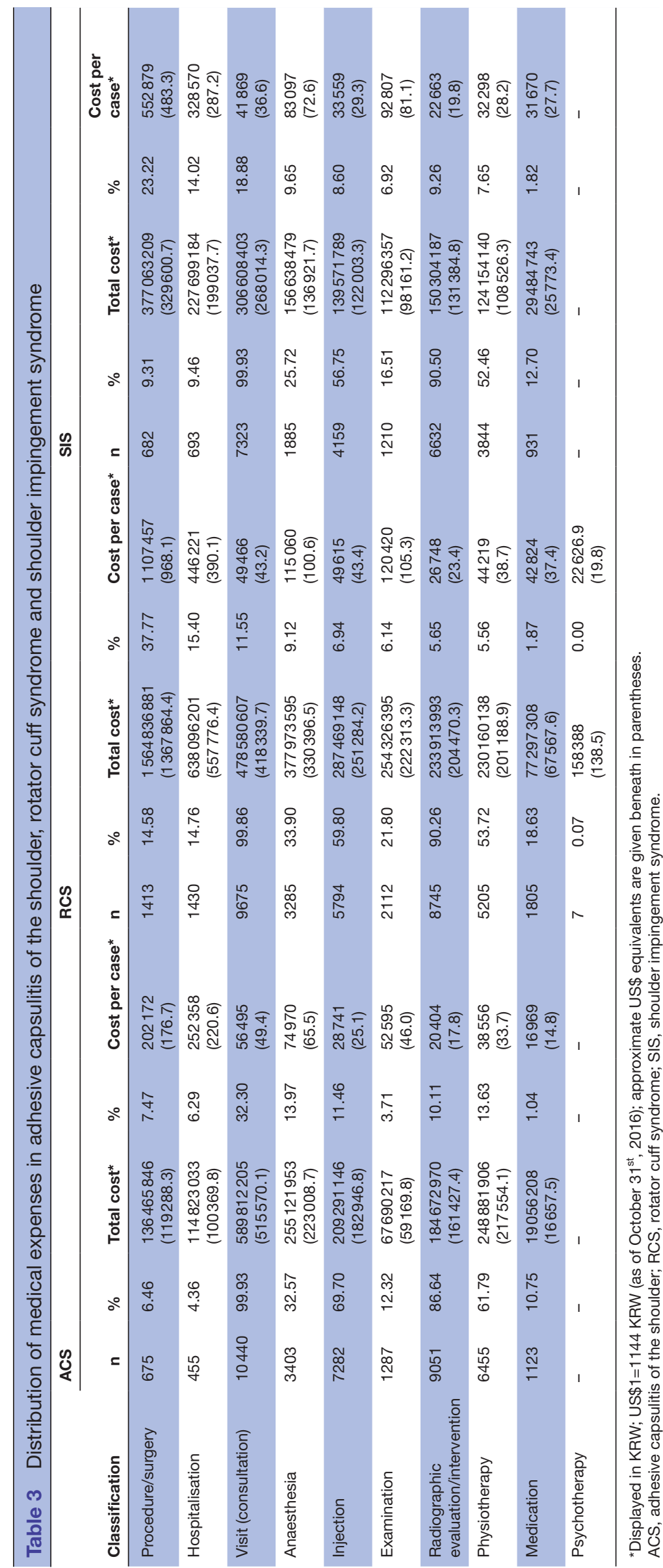




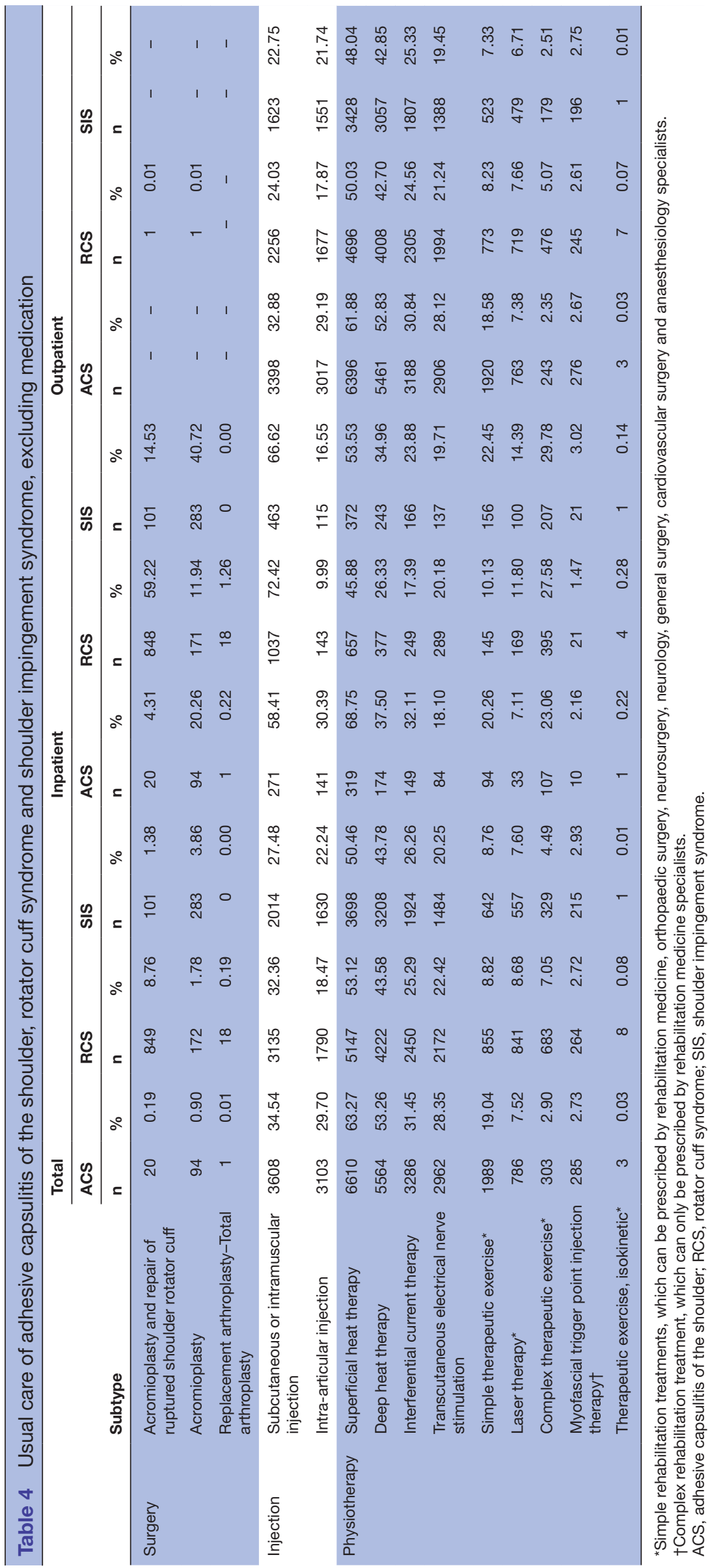




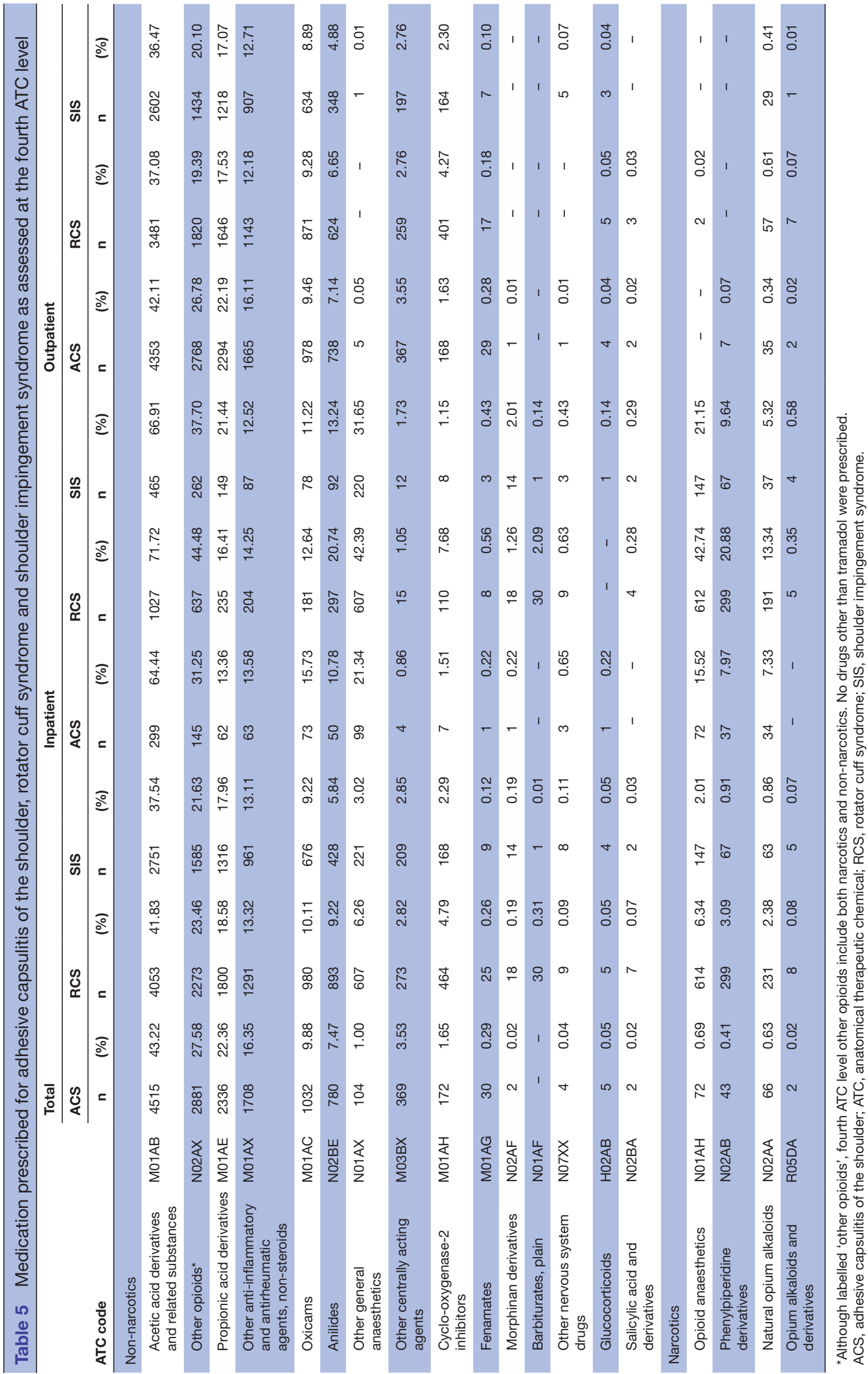


patients sought medical care from orthopaedic surgeons in all three groups, displaying marked specialty preference. The cost for visits (consultations) was largest in total medical expenses for the ACS and SIS groups, while cost for procedure/surgery was the predominant factor for patients with RCS. In all three groups, injection usage proportions were higher than those for drug prescription with a slightly higher injection percentage in patients with ACS. In physiotherapies, superficial heat therapy, deep heat therapy, transcutaneous electrical nerve stimulation and interferential current therapy were commonly used. Acetic acid derivatives and related substances were the most frequently prescribed non-narcotic analgesics out of fourth ATC levels, and opioid anaesthetics were the most frequently prescribed narcotics.

In a previous study on prevalence of shoulder disorders, ${ }^{4}$ supraspinatus tendinitis was the most common shoulder joint condition found in adults aged $\geq 40$ years, and prevalence of rotator cuff tear without trauma and adhesive capsulitis increased steeply with age, which is consistent with current study findings. Also, a number of studies have reported gender difference in prevalence of shoulder disorders, ${ }^{2417}$ and suggested explanations include hypotheses that women are more exposed to musculoskeletal risk factors in the form of various biological, sociological and psychological factors (eg, repetitive housework, lower accessibility to medical services and variance in sensory pain). ${ }^{618-20}$

Medical expenses were higher in inpatients than in outpatients for all three shoulder disorders, which is suggestive of sizeable economic burden from surgery. Total per-patient medical expenses were particularly high in the RCS group at twice the amount of the other two groups and may partially be explained by the high number of inpatients and surgically treated cases in this group. In light of these findings, medical policies pertaining to RCS should focus on hospitalisation in order to effectively address its socioeconomic cost.

Steady increase in surgical interventions for rotator cuff injury has been reported in the USA, ${ }^{21-23} \mathrm{UK}^{24}$ Denmark $^{25}$ and Finland ${ }^{26}$ in recent decades but has also been implicated with escalating medical expenditure. ${ }^{27} 28$ A 2008 Cochrane review failed to find conclusive evidence supporting surgical approaches for rotator cuff injuries, citing three independent trials reporting that therapeutic benefit of intensive rehabilitation programs were comparable to arthroscopic or subacromial decompression surgeries. ${ }^{29}$ In light of these results, coupled with the significant costs of surgery, surgical interventions should be considered when definite indications are clearly present. ${ }^{30}$

Notably, usage proportions of injection exceeded those of drug prescription in all three groups. Glenohumeral corticosteroid injection without guide imaging was previously shown to be more effective than oral non-steroidal anti-inflammatory drugs, and injections have been advocated over oral medicine with corticosteroids recommended for management of adhesive capsulitis on the grounds of fewer complications and adverse effects. ${ }^{31}$

$\mathrm{X}$-rays are regularly performed for diagnosis of most musculoskeletal conditions in Korea, including those of the shoulder joint. Shoulder radiographic findings are of particular use in OA, calcification (with sudden increase of pain), subacromial spurs (in clinically significant impingement), humeral head elevation and narrowing of the subacromial space. ${ }^{32}$

This study results show that heat and electrical therapies were the most popular physiotherapies for shoulder disorders in Korea. Previous studies report that the evidence for the effect of electrical therapies in treatment of such disorders as subacromial impingement syndrome or rotator cuff tendinitis is unclear when compared with placebo. ${ }^{33}$ Although the treatment failed to accelerate recovery, it is assumed that it continues to be widely used in clinical settings because of its temporary pain-relieving effects and low adverse event occurrence. These findings are comparable and compatible with National Health Insurance claims data low back pain (LBP) analysis results where heat and electrical therapies were consistently used at similar frequencies and distribution regardless of LBP type. ${ }^{35}$ It can be inferred that heat and electrical therapies are widely used as usual care of various musculoskeletal diseases relating to shoulder pain and LBP in Korea despite lack of supporting evidence. Simple therapeutic exercise tended to be prescribed more often in patients with ACS (19.04\%) than in patients with RCS $(8.82 \%)$ or patients with SIS $(8.76 \%)$. Griggs $e t a l^{36}$ reported in a prospective study of four shoulder stretching exercise regimens in 75 patients with phase II shoulder impingement that exercise led to improved pain, range of motion (ROM) and functionality as measured by Disabilities of the Arm, Shoulder, and Hand in $90 \%$ of cases. Dierks et al also proposed gentle exercises that do not cause or exacerbate pain improve shoulder ROM. ${ }^{37}$

In Korea, certain types of physiotherapy and exercise therapy can only be prescribed by rehabilitation medicine specialists. For example, laser therapy and therapeutic exercise are classified as simple rehabilitation therapy, while myofascial trigger point injection therapy is considered complex rehabilitation therapy as it is perceived to require more specific medical attention. The low prescription proportion of myofascial trigger point injection therapy, laser therapy and therapeutic exercise may be partially explained by the fact that prescription is exclusively available to select specialists.

Ninety-seven per cent of all Koreans are enrolled in National Health Insurance, and claims data are widely accepted to be representative of the Korean population at a national level. The NPS dataset, managed and maintained by HIRA, contains detailed information on diagnosis, medical history, insurance payment and patient demographics. The current study employed HIRA-NPS data to investigate current medical usage, usual care and related expenditure in management of major shoulder disorders in Korea. As the HIRA-NPS data were extracted 
as a $3 \%$ sample of total claims data, number of patients and cost estimations for the total population may be gauged through multiplication by the weight value of 33.3. The present findings on medical care and costs for high prevalence shoulder conditions may be of use to physicians in building a basic structured foundation for standardised care.

The data analysed in the current study were based on nationwide medical institution diagnosis codes classified by Statistics Korea, which may lack precision in exclusively selecting shoulder symptom patients. In an attempt to overcome such limitations, only data for main diagnosis of shoulder disorders were collected, excluding subdiagnosis of shoulder lesions. However, such arbitrary exclusion of subdiagnosis groups may diminish overall representativeness of findings. Also, due to the fact that analysis was performed using administrative codes, and not actual clinical conditions or symptoms, the possibility of mismatched coding cannot be ruled out. Although clear diagnostic criteria exist in primary care for shoulder pain, SIS, supraspinatus tendonitis, biceps tendonitis and $\mathrm{ACS},{ }^{38}$ difference arising from such factors as individual preference, working policies, proficiency and errors must be taken into account in data conformity. Park $e t$ al purported that diagnosis processes tended to be more accurate in hospitalised or severe patients than in ambulatory or mild cases and in tertiary hospitals compared with primary care clinics. ${ }^{39}$ As the main objective of this study was to assess the current status of medical service use and usual care in patients with a main complaint of shoulder disorders, the inclusion criteria were initially set as patients with a primary diagnostic code for shoulder disorders of KCD codes. However, there was expert opinion that KCD codes are primarily an administrative coding system and that primary and secondary diagnosis KCD codes may not be clearly differentiated under current Korean healthcare conditions. Shoulder region $\mathrm{X}$-ray results were included as inclusion criteria to improve precision in patient identification as X-rays are routinely conducted in clinical practice in Korea to heighten accuracy in shoulder pathology diagnosis and treatment. This selection method is in line with the inclusion of lumbar $\mathrm{X}$-rays in defining patients with chronic LBP in a report using the same data source as the current study, issued by the National Evidence-based Healthcare Collaborating Agency, a government-run research entity. ${ }^{16}$ However, although the aim was to improve precision in identification of main diagnosis of shoulder disorders by including shoulder region X-rays, this also leads to exclusion of patients with shoulder disorder without X-ray results, which may be considered an additional limitation of this study.

Intrinsic limitations regarding current data exist in that information on individual patient income, educational levels, residential areas, serological findings, smoking status and drinking habits are lacking, which limits further investigation for causal relationships. Also, details on medical services including surgery, injection, physiotherapy, medication and other data that were clearly non-associated with shoulder disorders were discarded in the process of data identification and selection. However, whether the medical services included for analysis were exclusively provided for shoulder disorders could not be verified.

Finally, the HIRA-NPS dataset only includes data on items covered by National Health Insurance, and medical treatments or medications that are not covered by national insurance and drugs or supplements that are sold over-the-counter could therefore not be included in the present analysis, limiting appreciation of the full spectrum of usual care for shoulder disorder management in Korea.

The study results may be considered to hold significance largely in the following aspects: analysis of prevalence, patient characteristics, medical expenditure and specific medical service use of high-frequency shoulder disorders in Korea from nationally representative data sources may be used in establishing healthcare policies, appropriating budgets for shoulder disorders and providing general guidelines for high-frequency treatments (usual care) and related medical expenses of high-prevalence shoulder disorders-including medication use-to practitioners. Also, from a research standpoint, these findings act as a basic guide in clinical study design as pragmatic studies on shoulder disorders often set standard care or usual care as the control. The results further offer a window into current usual care for shoulder disorders in clinical settings from a healthcare data source representative of Korea, allowing comparisons to be drawn and thus holding international implications for clinicians, researchers and healthcare policy makers.

\section{CONCLUSION}

The prevalence, medical service use and usual care employed for shoulder disorder management in Korea were investigated through analysis of National Health Insurance claims data, as extracted and provided by HIRA. As observational studies on healthcare use in patients with shoulder disorder at a national level have not been conducted, the current report on cost distribution of common medical services may be used as basic data in policy-making processes. In addition, the detailed frequency analysis data of individual medical service use may prove useful for practitioners and researchers engaging in real-world practice and related studies.

Contributors HJ, J-SS, JL, YP, YKS and J-HC conceptualised the study. YJL, M-rK, WK and $\mathrm{I}-\mathrm{HH}$ acquired and analysed the data. HJ, YJL, M-rK, WK and I-HH drafted the manuscript. YJL, J-SS, JL, YP, YKS and J-HC critically revised the manuscript. YJL made valuable suggestions in the revision process. All authors gave approval of the final submitted version.

Funding This research was supported by a grant of the Korea Health Technology R\&D Project through the Korea Health Industry Development Institute (KHIDI), funded by the Ministry of Health \& Welfare, Republic of Korea (grant number: HB16C0011).

Competing interests None declared. 
Patient consent Written informed consent was not obtained from participants as the current sample dataset was extracted from Korean National Health Insurance claims data and made publicly available for research purposes by the Health Insurance Review and Assessment Service (HIRA). All data were removed of identifying personal information by HIRA prior to analysis in this study and therefore remained anonymous.

Ethics approval The present study was reviewed and approved by the Institutional Review Board of Jaseng Hospital of Korean Medicine (JASENG 201611002). Written informed consent was not obtained from participants as the current sample dataset was extracted from Korean National Health Insurance claims data and made publicly available for research purposes by the Health Insurance Review and Assessment Service (HIRA). All data were removed of identifying personal information by HIRA prior to analysis in this study and therefore remained anonymous.

Provenance and peer review Not commissioned; externally peer reviewed.

Data sharing statement HIRA data are third-party data not owned by the authors. Raw data are made available from the Health Insurance Review and Assessment Service (HIRA) in Korea through visit or mail upon direct, email or fax submission of the dataset request form and declaration of data use (http://opendata.hira.or. $\mathrm{kr} / \mathrm{home} . \mathrm{do}$ ) and payment of the transfer of data request fee (300 $000 \mathrm{KRW}$ per dataset).

Open Access This is an Open Access article distributed in accordance with the Creative Commons Attribution Non Commercial (CC BY-NC 4.0) license, which permits others to distribute, remix, adapt, build upon this work non-commercially, and license their derivative works on different terms, provided the original work is properly cited and the use is non-commercial. See: http://creativecommons.org/ licenses/by-nc/4.0/

(c) Article author(s) (or their employer(s) unless otherwise stated in the text of the article) 2017. All rights reserved. No commercial use is permitted unless otherwise expressly granted.

\section{REFERENCES}

1. Virta L, Joranger P, Brox Jl, et al. Costs of shoulder pain and resource use in primary health care: a cost-of-illness study in Sweden. BMC Musculoskelet Disord 2012;13:17.

2. Linsell L, Dawson J, Zondervan K, et al. Prevalence and incidence of adults consulting for shoulder conditions in UK primary care; patterns of diagnosis and referral. Rheumatology 2006;45:215-21.

3. van der Windt DA, Koes BW, de Jong BA, et al. Shoulder disorders in general practice: incidence, patient characteristics, and management. Ann Rheum Dis 1995;54:959-64.

4. Luime JJ, Koes BW, Hendriksen IJ, et al. Prevalence and incidence of shoulder pain in the general population; a systematic review. Scand J Rheumatol 2004;33:73-81.

5. National Health Insurance Service. Press release [Internet] Seoul. 2012 http://www.nhis.or.kr/portal/site/main/MENU_WBDCC01/ (accessed Jun 2014).

6. Urwin M, Symmons D, Allison T, et al. Estimating the burden of musculoskeletal disorders in the community: the comparative prevalence of symptoms at different anatomical sites, and the relation to social deprivation. Ann Rheum Dis 1998;57:649-55.

7. Charles J, Britt H, Fahridin S. Chronic musculoskeletal problems managed in general practice. Aust Fam Physician 2007;36:392.

8. Walker-Bone K, Palmer KT, Reading I, et al. Prevalence and impact of musculoskeletal disorders of the upper limb in the general population. Arthritis Rheum 2004;51:642-51.

9. Jones J, Hodgson J, Clegg T, et al. Self-reported work-related illness in 1995: results from a household survey. Sudbury, UK: HSE Books, 1998.

10. Meislin RJ, Sperling JW, Stitik TP. Persistent shoulder pain: epidemiology, pathophysiology, and diagnosis. Am J Orthop 2005;34(12 Suppl):5-9.

11. Kim J, Son M. National health insurance statistical yearbook. Seoul: Health Insurance Review and Assessment Service and National Health Insurance Service, 2014.

12. Sohn M, Jung M. Effects of public and private health insurance on medical service utilization in the National Health Insurance System: national panel study in the republic of Korea. BMC Health Serv Res 2016;16:503.
13. Kim L, Kim JA, Kim S. A guide for the utilization of Health Insurance Review and Assessment Service national patient samples. Epidemiol Health 2014;36:e2014008.

14. World Health Organization, Ministry of Health and Welfare. Health Service delivery Profile. Republic of Korea, 2012.

15. Ministry of Health and Welfare. Expenditure of insured items by National Health Insurance. 2015 http://opendata.hira.or.kr/op/opc/ olapMdcICaAsisStatsInfo.do (accessed Mar 2016).

16. Lee SM, Han SK, Kim JH, et al; Clinical effectiveness of injection therapy for chronic low back pain: Korean National Evidence-based Health Care Collaborating Agency, 2010:1-232.

17. Picavet HS, Schouten JS. Musculoskeletal pain in the Netherlands: prevalences, consequences and risk groups, the DMC(3)-study. Pain 2003;102:167-78.

18. Wijnhoven HA, de Vet HC, Picavet HS. Prevalence of musculoskeletal disorders is systematically higher in women than in men. Clin J Pain 2006;22:717-24.

19. Njalsson T, Sigurdsson JA, McAuley RG. Health problems in family practice. an icelandic multicentre study. Scand J Prim Health Care 1996;14:4-12.

20. Jordan KP, Kadam UT, Hayward R, et al. Annual consultation prevalence of regional musculoskeletal problems in primary care: an observational study. BMC Musculoskelet Disord 2010;11:144.

21. Vitale MA, Arons RR, Hurwitz S, et al. The rising incidence of acromioplasty. J Bone Joint Surg Am 2010;92:1842-50.

22. Ensor KL, Kwon YW, Dibeneditto MR, et al. The rising incidence of rotator cuff repairs. J Shoulder Elbow Surg 2013;22:1628-32.

23. Colvin AC, Egorova N, Harrison AK, et al. National trends in rotator cuff repair. J Bone Joint Surg Am 2012;94:227-33.

24. Judge A, Murphy RJ, Maxwell R, et al. Temporal trends and geographical variation in the use of subacromial decompression and rotator cuff repair of the shoulder in England. Bone Joint $J$ 2014;96B:70-4.

25. Svendsen SW, Frost P, Jensen LD. Time trends in surgery for nontraumatic shoulder disorders and postoperative risk of permanent work disability: a nationwide cohort study. Scand J Rheumatol 2012;41:59-65.

26. Paloneva J, Lepola V, Karppinen J, et al. Declining incidence of acromioplasty in Finland. Acta Orthop 2015;86:220-4.

27. Kuye IO, Jain NB, Warner L, et al. Economic evaluations in shoulder pathologies: a systematic review of the literature. J Shoulder Elbow Surg 2012;21:367-75.

28. lyengar JJ, Samagh SP, Schairer W, et al. Current trends in rotator cuff repair: surgical technique, setting, and cost. Arthroscopy 2014;30:284-8.

29. Coghlan JA, Buchbinder R, Green S, et al. Surgery for rotator cuff disease. Cochrane Database Syst Rev 2008;1:CD005619.

30. Hopman K, Krahe L, Lukersmith S, et al. Clinical practice guidelines for the management of rotator cuff syndrome in the workplace. Port Macquarie (Australia): University of New South Wales, 2013:80.

31. Ranalletta M, Rossi LA, Bongiovanni SL, et al. Corticosteroid injections accelerate pain relief and recovery of function compared with oral NSAIDs in patients with adhesive capsulitis: a randomized controlled trial. Am J Sports Med 2016;44:474-81.

32. Mashford L, Allen R, Barnsley L. Therapeutic guidelines: rheumatology. Melbourne: Therapeutic Guidelines Ltd, 2006.

33. Binder A, Parr G, Hazleman B, et al. Pulsed electromagnetic field therapy of persistent rotator cuff tendinitis. A double-blind controlled assessment. Lancet 1984;1:695-8.

34. Aktas I, Akgun K, Cakmak B. Therapeutic effect of pulsed electromagnetic field in conservative treatment of subacromial impingement syndrome. Clin Rheumatol 2007;26:1234-9.

35. Ahn YJ, Shin JS, Lee J, et al. Evaluation of use and cost of medical care of common lumbar disorders in Korea: cross-sectional study of Korean Health Insurance Review and Assessment Service National Patient Sample data. BMJ Open 2016;6:e012432.

36. Griggs SM, Ahn A, Green A. Idiopathic adhesive capsulitis. A prospective functional outcome study of nonoperative treatment. $J$ Bone Joint Surg Am 2000;82-A:1398-407.

37. Diercks RL, Stevens M. Gentle thawing of the frozen shoulder: a prospective study of supervised neglect versus intensive physical therapy in seventy-seven patients with frozen shoulder syndrome followed up for two years. J Shoulder Elbow Surg 2004;13:499-502.

38. House J, Mooradian A. Evaluation and management of shoulder pain in primary care clinics. South Med J 2010;103:1129-37.

39. Park B, Seong J, Park G, et al. Studying on improving diagnosis codes in National Health Insurance claims data. Seoul: Health Insurance Review and Assessment Service, 2003. 